\title{
microPET-Based Biodistribution of Quantum Dots in Living Mice
}

\author{
Meike L. Schipper ${ }^{1}$, Zhen Cheng ${ }^{1}$, Sheen-Woo Lee ${ }^{1}$, Laurent A. Bentolila ${ }^{2,3}$, Gopal Iyer ${ }^{3}$, Jianghong Rao ${ }^{1}$, \\ Xiaoyuan Chen ${ }^{1}$, Anna M. Wu ${ }^{4}$, Shimon Weiss ${ }^{2,3}$, and Sanjiv S. Gambhir ${ }^{1}$ \\ ${ }^{I}$ Departments of Radiology and Bioengineering, Bio-X Program, Molecular Imaging Program at Stanford (MIPS), Stanford University, \\ Stanford, California; ${ }^{2}$ California NanoSystems Institute (CNSI), UCLA School of Medicine, Los Angeles, California; ${ }^{3}$ Department of \\ Chemistry and Biochemistry, UCLA School of Medicine, Los Angeles, California; and ${ }^{4}$ Department of Molecular \& Medical \\ Pharmacology, Crump Institute for Molecular Imaging, UCLA School of Medicine, Los Angeles, California
}

This study evaluates the quantitative biodistribution of commercially available CdSe quantum dots (QD) in mice. Methods: ${ }^{64} \mathrm{Cu}$-Labeled 800 - or 525-nm emission wavelength QD (21- or 12-nm diameter), with or without 2,000 MW (molecular weight) polyethylene glycol (PEG), were injected intravenously into mice (5.55 MBq/25 pmol QD) and studied using well counting or by serial microPET and region-of-interest analysis. Results: Both methods show rapid uptake by the liver (27.4-38.9 \% ID/ g) $(\% \mathrm{ID} / \mathrm{g}$ is percentage injected dose per gram tissue) and spleen (8.0-12.4\%ID/g). Size has no influence on biodistribution within the range tested here. Pegylated QD have slightly slower uptake into liver and spleen (6 vs. $2 \mathrm{~min}$ ) and show additional low-level bone uptake (6.5-6.9 \% ID/g). No evidence of clearance from these organs was observed. Conclusion: Rapid reticuloendothelial system clearance of QD will require modification of QD for optimal utility in imaging living subjects. Formal quantitative biodistribution/imaging studies will be helpful in studying many types of nanoparticles, including quantum dots.

Key Words: quantum dot; biodistribution; nanoparticle; PET; molecular imaging

J Nucl Med 2007; 48:1511-1518

DOI: 10.2967/jnumed.107.040071

Q uantum dots (QD) are fluorescent semiconductor nanocrystals with high quantum yield, resistance to photobleaching, narrow emission peak, tunable emission wavelength, and constant excitation profile regardless of emission wavelength, making them interesting for in vivo smallanimal imaging $(1,2)$. Naturally hydrophobic, QD are made water-soluble by surface conjugation (1). Targeting molecules, such as antibodies (3), aptamers (4), peptides $(5,6)$, folate (7), or high-molecular-weight dextran (8) can then be added. Polyethylene glycol (PEG) is commonly attached to the surface of QD for in vivo applications. Pegylation

Received Jan. 24, 2007; revision accepted May 7, 2007.

For correspondence or reprints contact: Sanjiv S. Gambhir, MD, PhD, Molecular Imaging Program at Stanford, East 150 Clark Center, 318 Campus Dr., Palo Alto, CA 94305-5427.

E-mail: sgambhir@stanford.edu

COPYRIGHT @ 2007 by the Society of Nuclear Medicine, Inc. increases in vivo circulation times of nanoparticles and liposomes, likely by sterically hindering the absorption of opsonizing proteins and, thus, delaying recognition and clearance by the reticuloendothelial system (RES) $(9,10)$.

QD have been used for in vivo fluorescence imaging for fluorescence sentinel lymph node mapping (11-13), or diffusion analysis of the brain extracellular space (14), in which particle size confines QD to a specific compartment or clearance route. Other studies have used surface modifications to target QD. Akerman et al. used ex vivo fluorescence microscopy to show lung and tumor vasculature targeting of peptide- and 5,000 MW (molecular weight) PEG-coated CdSe/ZnS QD (5). Significant liver and spleen uptake of QD was noted, and it remains unclear whether tumor uptake in this study would have been sufficient for in vivo noninvasive fluorescence imaging. In vivo fluorescence imaging of targets expressed in tumor vasculature and tumor tissues has been reported by our group $\left(\alpha_{\mathrm{v}} \beta_{3}\right.$ integrin targeting using 15- to 20-nm-wide CdSe/ZnS QD coated with RGD peptide and 2,000 MW PEG (6), and others (prostate-specific membrane antigen [PSMA] targeting with 10- to 15 -nm-diameter CdSe/ZnS QD coated with anti-PSMA monoclonal antibodies and 5,000 MW PEG (3)). Both reports show significant QD uptake in liver and spleen, without quantifying uptake. Ballou et al. show clearance from circulation of untargeted 15- to 20-nm-diameter $\mathrm{CdSe} / \mathrm{ZnS}$ QD coated with $750 \mathrm{MW}$ PEG or $3,400 \mathrm{MW}$ PEG by liver and spleen within $12 \mathrm{~min}$, whereas 5,000 MW PEG QD were imaged for up to $140 \mathrm{~min}$ (15). Fluorescence imaging showed liver, spleen, bone marrow, and lymph nodes to be involved in QD clearance. However, tracking and quantification of QD in vivo by fluorescence imaging is limited by tissue absorption of light, which impairs excitation of QD in deeper lying tissues, and decreases fluorescent light penetration from deeper structures to the surface, where it can be measured (16). Despite the recent development of self-illuminating QD by our group (17) and continuing efforts to correct for tissue absorption with mathematic algorithms, to date, fluorescence cannot be measured in a fully quantitative way. The biodistribution of 
QD in living animals thus remains unclear, despite preliminary results from fluorescence imaging studies $(3,6,15)$.

Biodistribution studies provide crucial quantitative and temporal information of an imaging agent. They assess its usefulness for reaching and imaging the intended targetfor example, in the vasculature or on tumor cells. The information gathered helps the development of new or improved imaging probes and therapy agents. Traditional biodistribution studies using well counting require sacrifice of 50-60 animals per compound. The significant expense of lives, funds, and time limits the number of time points that can be assessed. In contrast, PET of small animals allows for serial imaging of live mice, obviates the need to sacrifice animals, and minimizes interindividual variation, resulting in better data quality (18). Imaging software can be used to measure radioactivity within regions of interest (ROIs) in the resulting datasets. Dynamic acquisition, or serial static imaging, allows for the acquisition of theoretically unlimited numbers of time points from one mouse.

The current study evaluated the biodistribution of commercially available CdSe QD. QD were radiolabeled with ${ }^{64} \mathrm{Cu}$ and studied both with conventional well counting and by serial microPET with ROI analysis. Larger (800-nm emission wavelength; 21-nm diameter) and smaller (525$\mathrm{nm}$ emission wavelength; 12-nm diameter) QD were studied. We further investigated whether surface coating with 2,000 MW PEG, which prolongs the circulation half-life of liposomes and other nanoscale particles, leads to an increased circulation half-life of QD.

\section{MATERIALS AND METHODS}

\section{QD}

Qdot 525 ITK amino (PEG) ("QD525PEG"), Qdot 800 ITK amino (PEG) (“QD800PEG”), Qdot 525 ITK carboxyl ("QD525"), and Qdot 800 ITK carboxyl ("QD800") CdSe/ZnS Core Shell Quantum Dots (QD), from Invitrogen, are coated with a proprietary polymer and covalently conjugated with 2,000 MW PEG, where applicable.

\section{Chemistry and Radiochemistry}

Unless otherwise mentioned, chemicals were obtained from Sigma-Aldrich Chemical Co. A CRC-15R PET dose calibrator (Capintec Inc.) was used. DOTA (1,4,7,10-tetraazacyclododecane1,4,7,10-tetraacetic acid; Macrocyclics Inc.) (for QD525PEG and QD800PEG) (or DOTA-lysine [for QD525 and QD800], respectively), 1-ethyl-3-[3-(dimethylamino)propyl-]carbodiimide (EDC), and $N$-hydroxysulfonosuccinimide (SNHS) at a molar ratio of 1:1:0.8 were mixed and incubated at $4^{\circ} \mathrm{C}$ for $30 \mathrm{~min}(\mathrm{pH} 5.5)$. QD $(8.2 \mu \mathrm{M}, 100 \mu \mathrm{L})$ were then reacted with the in situ prepared sulfosuccinimidyl ester of DOTA (DOTA-OSSu) in a ratio of $1: 1,000$ in $50 \mathrm{mM}$ sodium borate buffer $(\mathrm{pH} 8.4)$ at $4^{\circ} \mathrm{C}$ overnight. The resulting DOTA-PEG-QD [DOTA-QD] were purified by centrifuge filtration $10 \mathrm{~K}$ nanosep (Pall Corp.).

DOTA-PEG-QD [DOTA-QD] $(4.1 \mu \mathrm{M}, 20 \mu \mathrm{L})$ were radiolabeled with $44.4 \mathrm{MBq}{ }^{64} \mathrm{CuCl}_{2}$ (University of Wisconsin-Madison, Madison, WI) in $0.1 \mathrm{~N} \mathrm{NaOAc}\left(\mathrm{pH} \mathrm{5.5)}\right.$ for $1 \mathrm{~h}\left(37^{\circ} \mathrm{C}\right)$, purified (10K Nanosep), and reconstituted in phosphate-buffered saline (PBS). Radiolabeling yield was $\geq 95 \%$, and specific activity was estimated to be $\geq 37 \mathrm{GBq} / \mu \mathrm{mol}$ for all radiolabeled complexes, equivalent to one ${ }^{64} \mathrm{Cu}$-labeled QD in $100 \mathrm{QD}$. Administration of unlabeled QD was considered to be an advantage because it allowed for administration of $5.55 \mathrm{MBq}$ in 25 pmol QD per animal-a QD dose that is commonly used by our group for fluorescence imaging (Meike L. Schipper, Olivier Gheysens, unpublished data, August 2006).

\section{Biodistribution}

All animal experiments were performed in accordance with protocols approved by the Stanford Administrative Panel on Laboratory Animal Care. Eight- to 12-wk-old nude mice (Charles River) were tail vein-injected with 25 pmol of ${ }^{64} \mathrm{Cu}$-DOTAlabeled (1.85 MBq) QD525PEG or QD800PEG in $150 \mu \mathrm{L}$ of PBS, respectively. Three animals per group were sacrificed at $10 \mathrm{~min}$, $30 \mathrm{~min}, 1.5 \mathrm{~h}, 4.5 \mathrm{~h}, 12 \mathrm{~h}$, or $36 \mathrm{~h}$, respectively. Tissues were harvested, weighed, and counted for $1 \mathrm{~min}$ in a Cobra II $\gamma$-counter (Packard/Perkin Elmer). Results are expressed as the percentage injected dose per gram tissue (\%ID/g).

\section{microPET}

Nude mice were imaged under anesthesia with $2 \%$ isoflurane in $2 \mathrm{~L} / \mathrm{min}$ of oxygen on a microPET R4 scanner (Siemens Medical Solutions USA, Inc.). Image acquisition was initiated before tail vein injection of $5.55 \mathrm{MBq}$ of ${ }^{64} \mathrm{Cu},{ }^{64} \mathrm{Cu}$-DOTA, ${ }^{64} \mathrm{Cu}$-DOTAQD525, ${ }^{64} \mathrm{Cu}$-DOTA-QD800, ${ }^{64} \mathrm{Cu}$-DOTA-QD525PEG, or ${ }^{64} \mathrm{Cu}-$ DOTA-QD800PEG, respectively. Dynamic 10-s time frames were acquired for $10 \mathrm{~min}$ after injection, followed by one 5-min time frame. Animals were then reimaged at $60 \mathrm{~min}, 4.5 \mathrm{~h}, 12 \mathrm{~h}$, and $36 \mathrm{~h}$ after injection, using 5-min static acquisitions. Images were reconstructed using an ordered-subset expectation maximization (OSEM) algorithm. The final image spatial resolution is 1.66-1.85 $\mathrm{mm}(19)$.

\section{MicroCT}

Immediately after microPET, nude mice were scanned on an eXplore RS-9 MicroCT System (GE Healthcare) under anesthesia with $2 \%$ isoflurane in $2 \mathrm{~L} / \mathrm{min}$ of oxygen for $6 \mathrm{~min}$ in 2 bed positions. Acquisition parameters were as follows: $70-\mathrm{kVp}$ beam energy, $40-\mu \mathrm{A}$ current, $4.2-\mathrm{cm}$ field of view (z-axis), and 200 projections. EXplore Evolver and eXplore Reconstruction Interface software was used for image acquisition and reconstruction. Images were viewed using GE Healthcare Microview and AMIDE. The spatial resolution of the images was isotropic at $97 \mu \mathrm{m}$.

\section{Image-Based In Vivo Biodistribution}

Reconstructed images were loaded into AMIDE, a free image analysis software package developed in our laboratories (20). CT and microPET datasets of each individual animal were manually aligned to ensure good overlap of organs of interest. Threedimensional ROIs were placed within brain, heart, lung, liver, spleen, kidneys, bladder, gut, testes, muscle, and bone, using morphologic CT information to delineate organs with low uptake, and functional PET information for organs with high uptake. Activity per voxel was converted to $\% \mathrm{ID} / \mathrm{g}$ using conversion factors obtained by scanning a cylindric phantom filled with a known activity of ${ }^{64} \mathrm{Cu}$ to account for microPET scanner efficiency (16). The \% ID/g of each organ for the midpoint of each frame, or time point, respectively, was then plotted over time. 


\section{Statistical Calculations}

Averages, SD, and statistical significance ( $t$ test) were determined using standard software (Excel 2000; Microsoft Corp.). $P$ values $<0.05$ were considered statistically significant.

\section{RESULTS}

\section{Ex Vivo Biodistribution Shows Rapid Uptake and Retention in Liver and Spleen}

Ten minutes after injection, liver and spleen were organs of major QD accumulation (Fig. 1). Liver accumulated $29.6 \pm 8.6$ and $54.5 \pm 11.0 \% \mathrm{ID} / \mathrm{g}$ of ${ }^{64} \mathrm{Cu}$-labeled QD525PEG and QD800PEG, respectively, whereas spleen took up $12.2 \pm 7.4$ and $37.2 \pm 13.4 \% \mathrm{ID} / \mathrm{g}$ of ${ }^{64} \mathrm{Cu}-$ labeled QD525PEG and QD800PEG. Femur and lung accumulated small amounts of QD as well (3.8 \pm 5.2 and $4.3 \pm 2.0 \% \mathrm{ID} / \mathrm{g}$ of QD525PEG and QD800PEG [femur] and $3.7 \pm 1.0$ and $1.4 \pm 1.6 \% \mathrm{ID} / \mathrm{g}$ of QD525PEG and QD800PEG [lung], respectively). All other organs showed negligible uptake $10 \mathrm{~min}$ after injection.

The pattern remained roughly identical throughout the remainder of the study. Absolute values of uptake in liver varied $(35.7 \pm 8.6$ [at $30 \mathrm{~min}$ ] to $47.1 \pm 11.5$ [at $4.5 \mathrm{~h}$ ] $\% \mathrm{ID} / \mathrm{g}$ for QD525PEG and $31.6 \pm 17.2$ [at $30 \mathrm{~min}$ ] to $53.1 \pm$
9.4 [at $4.5 \mathrm{~h}$ ] \% ID/g for QD800PEG). However, liver uptake at $10 \mathrm{~min}$ was not significantly different from uptake at $35 \mathrm{~h}$ (QD525PEG $[P>0.1]$ and QD800PEG $[P>0.6])$ if physical decay of the radiolabel was accounted for. Likewise, splenic uptake varied (7.5 \pm 3.2 [at $12 \mathrm{~h}$ ] to $22.3 \pm 12.6$ [at $30 \mathrm{~min}$ )] $\% \mathrm{ID} / \mathrm{g}$ for QD525PEG and $12.5 \pm 6.5$ [at $1.5 \mathrm{~h}$ ] to $26.5 \pm 4.7$ [at $12 \mathrm{~h}$ ] $\% \mathrm{ID} / \mathrm{g}$ for QD800PEG) but did not differ significantly between $10 \mathrm{~min}$ and $35 \mathrm{~h}$ if physical decay was considered $(P>0.2$ and $P>0.08$ for QD525PEG and QD800PEG, respectively). With the exception of lung, femur, and lymph node in QD525PEG and lung and femur in QD800PEG, activity in all other organs remained low (Fig. 1).

\section{microPET-Based In Vivo Biodistribution of Pegylated QD800 and QD525 Shows Rapid and Predominant Uptake in Liver, Spleen, and Bone}

Dynamic imaging revealed a brief initial peak in heart and lung uptake, as expected from a transient increase in blood-pool activity. As previously found in the ex vivo biodistribution study, liver was the organ of predominant uptake for both QD525PEG and QD800PEG, followed by spleen, and bone (Fig. 2; supplemental videos 1-6 are
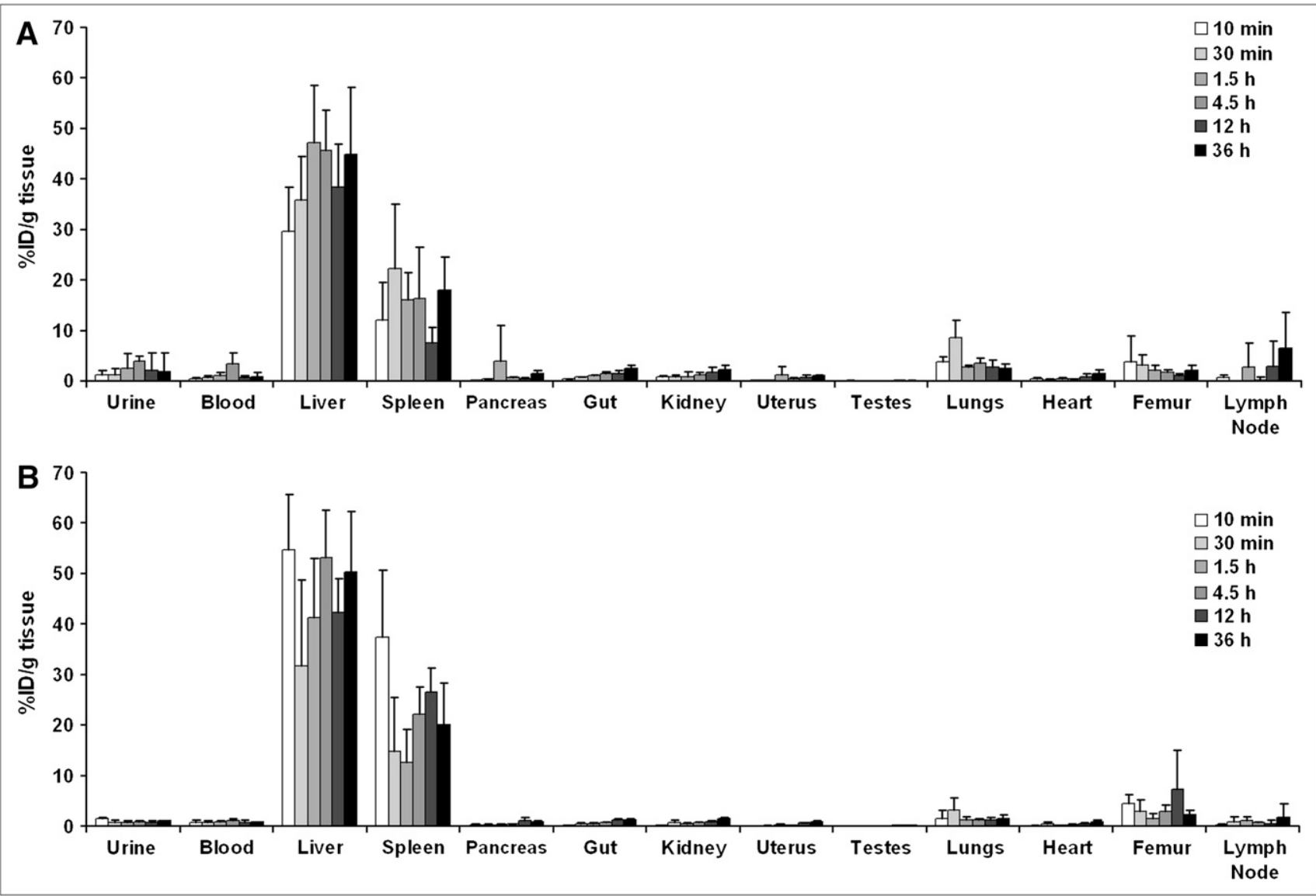

FIGURE 1. Ex vivo biodistribution of ${ }^{64} \mathrm{Cu}$-labeled, pegylated QD525 (A) and QD800 (B) as measured by well counting. Radiolabeled QD (1.85 MBq) were injected into tail vein of nude mice. Groups of mice $(n=3)$ were sacrificed at 10 min, 30 min, $1.5 \mathrm{~h}, 4.5 \mathrm{~h}, 12 \mathrm{~h}$, and $36 \mathrm{~h}$, and organs were harvested and counted. Mean and SD of \%ID/g have been corrected for physical decay of isotope. 


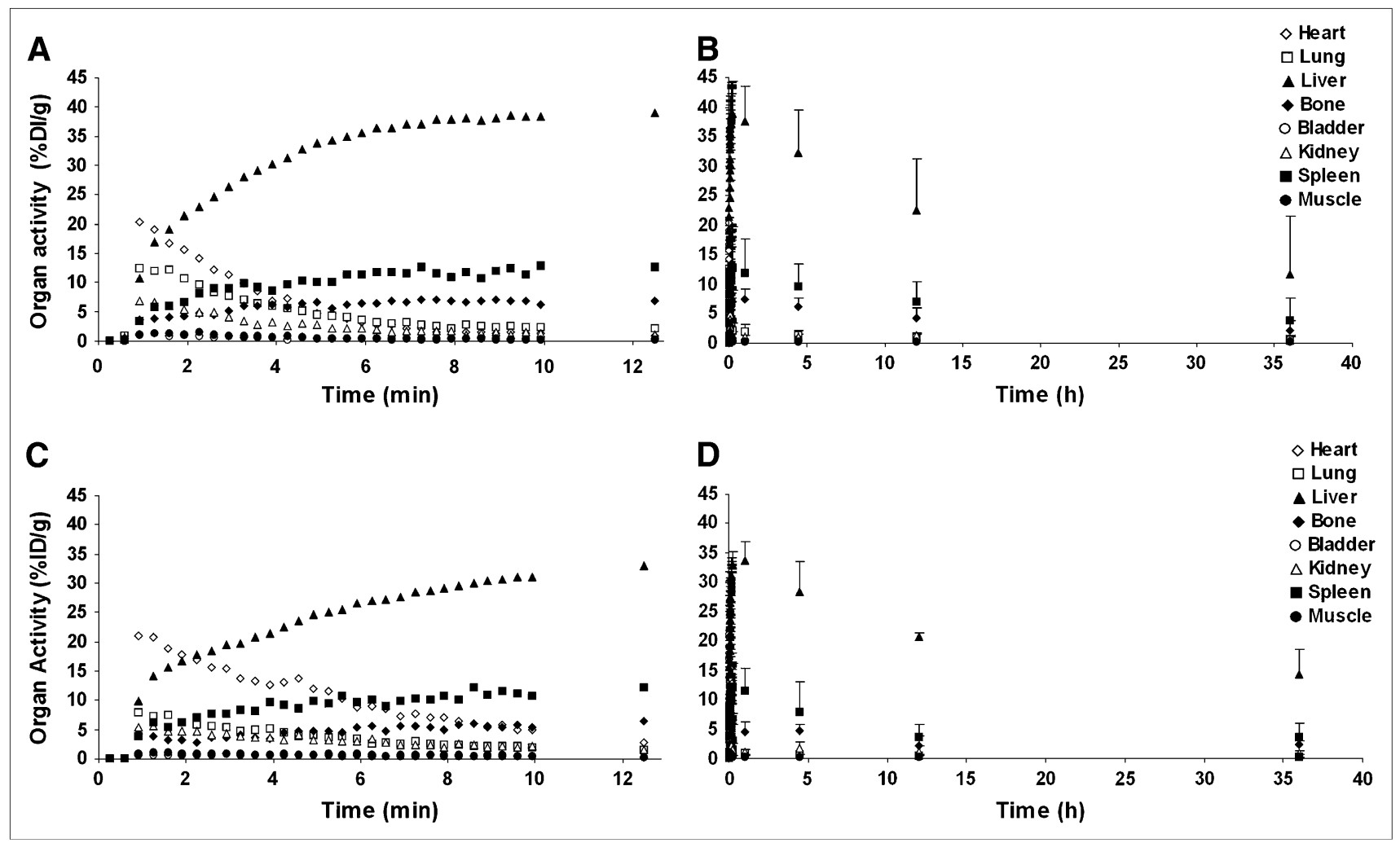

FIGURE 2. Image-based in vivo biodistribution of ${ }^{64}$ Cu-labeled, pegylated QD525 (A and B) and QD800 (C and D) as measured by image ROI analysis of microPET datasets. During dynamic image acquisition, $5.55 \mathrm{MBq}$ of radiolabeled QD were injected into tail vein of nude mice $(n=4)$. Images were acquired dynamically in 10-s frames for the first 10 min and one 5-min frame thereafter (A and C; error bars are omitted for better visibility). Mice were reimaged in 5-min static acquisitions at 1, 3, 6, 12, and 36 h (B and D), coregistered with microCT images, and AMIDE image analysis software was used to obtain organ activity information. Mean and SD of \% ID/g have not been corrected for physical decay.

available online only at http://jnm.snmjournals.org; see supplemental videos 1 and 2). Within 6 min of injection, liver activity levels were within $1 \mathrm{SD}$ of their maximum $(38.9 \pm 5.5$ and $32.9 \pm 2.2 \% \mathrm{ID} / \mathrm{g}$ for QD525PEG and QD800PEG, respectively; Fig. 2). Following similar kinetics, spleen accumulated $12.6 \pm 7.1$ and $12.3 \pm 4.0 \% \mathrm{ID} / \mathrm{g}$ of QD525PEG and QD800PEG, and bone took up $6.9 \pm$ 2.0 and $6.5 \pm 1.2 \% \mathrm{ID} / \mathrm{g}$ of QD525PEG and QD800PEG. Other organs showed negligible uptake from $10 \mathrm{~min}$ after injection throughout the remainder of the study (Fig. 3, fourth and sixth columns). Liver, spleen, and bone uptake did not decrease significantly over the time of the study if physical decay of the radiolabel was accounted for (Fig. 2).

\section{Unpegylated QD800 and QD525 Are Taken Up Immediately by Liver and Spleen But Not by Bone}

After an initial rapid peak, blood-pool activity decreased to background levels even faster than that seen with pegylated QD. Liver and spleen activity reached nearmaximal levels within 2 min (Fig. 4; supplemental videos 3 and 4). Maximum liver and spleen activities were slightly lower than those in pegylated QD (liver: $27.4 \pm 2.2$ and $27.4 \pm 6.1 \% \mathrm{ID} / \mathrm{g}$ of QD525 and QD800, respectively; and spleen: $8.7 \pm 2.7$ and $8.0 \pm 7.0 \% \mathrm{ID} / \mathrm{g}$ of QD525 and QD800, respectively). Interestingly, unpegylated QD showed no relevant bone uptake $(1.5 \pm 0.4$ and $1.7 \pm 0.4 \% \mathrm{ID} / \mathrm{g}$ of QD525 and QD800, respectively, at $10 \mathrm{~min}$ ) (Fig. 3, third and fifth columns). As previously observed, other organs showed background uptake of unpegylated QD throughout the study, and decreases in liver and spleen uptake were explained by physical decay of the radiolabel (Fig. 4).

\section{DOTA-Chelated ${ }^{64} \mathrm{Cu}$ Is Rapidly Excreted Through the Kidneys, Whereas Free ${ }^{64} \mathrm{Cu}$ Is Accumulated by Liver and Spleen in Addition to Renal Excretion}

To assess the stability of ${ }^{64} \mathrm{Cu}$-DOTA-labeled QD in vivo, we performed biodistribution studies for both DOTAcomplexed ${ }^{64} \mathrm{Cu}$ and free ${ }^{64} \mathrm{Cu}$ (Fig. 5; supplemental videos 5 and 6). Following blood-pool activity peaks in heart and lung activity, these tracers displayed marked differences in biodistribution patterns. DOTA-chelated ${ }^{64} \mathrm{Cu}$ was rapidly accumulated by the kidney, peaking around $80 \mathrm{~s}$ after injection $(53.9 \pm 24.5 \% \mathrm{ID} / \mathrm{g})$ and excreted into the bladder (maximum activity at $15 \mathrm{~min}, 402.3 \pm 297.5 \% \mathrm{ID} / \mathrm{g}$ ). Liver, spleen, and bone do not accumulate relevant amounts of DOTA-chelated ${ }^{64} \mathrm{Cu}(1.2 \pm 0.2,0.9 \pm 0.3$, and $0.9 \pm$ $0.2 \% \mathrm{ID} / \mathrm{g}$, respectively, at $15 \mathrm{~min}$ ), just like all other organs (Fig. 3, second column). At 1 h, kidney clearance of DOTA-chelated ${ }^{64} \mathrm{Cu}$ has eliminated relevant activity from all organs except the bladder. Similar to QD, free ${ }^{64} \mathrm{Cu}$ is 


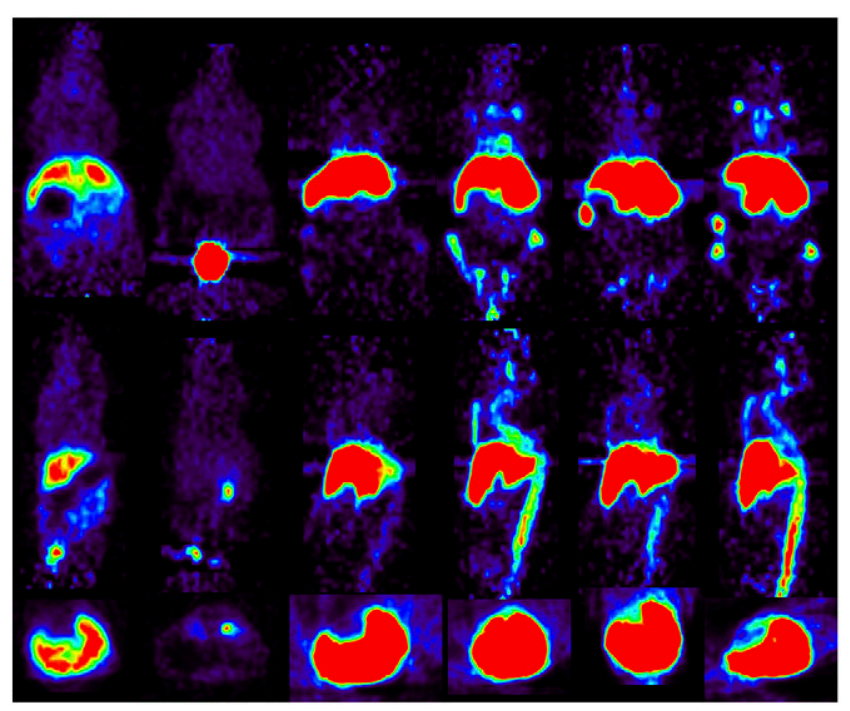

FIGURE 3. In vivo PET images of mice injected with ${ }^{64} \mathrm{Cu}$ (first column), ${ }^{64} \mathrm{Cu}-\mathrm{DOTA}$ (second column), QD525 (third column), QD525PEG (fourth column), QD800 (fifth column), or QD800PEG (sixth column). During dynamic image acquisition, $5.55 \mathrm{MBq}$ of the respective agent were injected into tail vein of nude mice. Images were acquired dynamically in 10-s frames for the first $10 \mathrm{~min}$ and one 5-min frame thereafter. Coronal (upper row), sagittal (middle row), and transverse (lower row) slices of a 5-min frame from 10 to $15 \mathrm{~min}$ after injection are shown.

taken up by liver and spleen $(21.1 \pm 0.6$ [liver] and $4.7 \pm$ 2.3 [spleen] \% ID/g at $15 \mathrm{~min}$ ). In contrast to QD, however, a significant amount of free ${ }^{64} \mathrm{Cu}$ is accumulated by the kidneys (peaking at $10.5 \pm 0.9 \% \mathrm{ID} / \mathrm{g}$ around $80 \mathrm{~s}$ after injection) and excreted into the bladder $(10.0 \pm 1.9 \% \mathrm{ID} / \mathrm{g})$. All other organs have low uptake (Fig. 3, first column). Unlike that seen with ${ }^{64} \mathrm{Cu}$-DOTA-labeled QD, activity of free ${ }^{64} \mathrm{Cu}$ in the liver decreases faster than physical decay would suggest. The effective half-life of liver activity for free ${ }^{64} \mathrm{Cu}$ is $6.25 \mathrm{~h}$ (as opposed to the $12.9 \mathrm{~h}$ expected from physical decay), consistent with the presence of biologic clearance.

\section{DISCUSSION}

QD and other nanoscale materials hold great promise for biomedical applications such as tumor research. However, in any in vivo application, the potential usefulness of an agent cannot be assessed without knowledge of quantitative biodistribution. Currently, it is not possible to accurately quantify biodistribution from emitted light that is measured outside the animal. This is consistent with observations made by our laboratory comparing light output with PET biodistribution signal of radiolabeled QD (data not shown).

We studied the quantitative biodistribution of QD in living nude mice, a relevant animal model for tumor research. Commercially available QD formulations were cleared from the circulation within $10 \mathrm{~min}$ of tail-vein injection. Liver and spleen, the primary locations of the RES, are the primary organs of QD uptake, as shown by both traditional and image-based biodistribution. The likely mechanism, opsonization of QD by serum proteins-such as members of the complement and clotting cascade, and subsequent clearance by RES - has been extensively corroborated for other nanoparticles of similar size $(9,10,21)$. Surface modification with 2,000 MW PEG increased particle blood halflife from $20 \mathrm{~s}$ to $3-4 \mathrm{~min}$ but had little effect on overall kinetics, merely increasing the time for QD to accumulate in the liver from 2 to $6 \mathrm{~min}$. This finding appears discrepant with prior reports of blood circulation times up to $140 \mathrm{~min}$ obtained with 5,000 MW conjugated QD, as measured by fluorescence imaging of superficial vasculature (15). However, circulation half-life does not increase linearly with PEG chain length, but depends on a range of factors influencing PEG tertiary structure, including PEG chain length, PEG density, and surface potential of the underlying nanostructure $(9,10,21)$. Clearance may also be influenced by the amount of nanoparticles injected. Liposomes, which are shielded from the RES by 2,000 MW PEG coating, will be cleared rapidly from the blood when injected at doses of $<20 \mathrm{nmol} / \mathrm{kg}$. This phenomenon is attributed to a pool of preexisting opsonic factors in the blood, which is depleted as higher doses are given (22-24). In our study, mice were administered $25 \mathrm{pmol}$ of QD, or $0.8-1.25 \mathrm{nmol} / \mathrm{kg}$ (depending on weight of the animal), well below the concentration of liposomes reported to be cleared rapidly. Future studies will need to address whether a similar pattern of rapid lowdose clearance exists for QD and whether longer circulation half-lives can be achieved either by injecting more QD or preinjecting other agents that deplete any preexisting opsonins (baiting). Pegylation led to bone uptake, which was not seen with unpegylated QD. Likely, the increased temporal availability of nanoparticles in the blood enabled secondary RES locations, such as macrophages in bone marrow, to participate in the clearance process.

No difference in biodistribution was observed between larger (hydrodynamic diameter of $21 \mathrm{~nm}$ ) and smaller (hydrodynamic diameter of $12 \mathrm{~nm}$ ) QD. This may reflect an inability of both particles to spontaneously cross the endothelial cell/basement membrane barrier of the blood vessel in the time of their presence in the circulation. Indeed, Stroh et al. did not observe any extravasation of QD from tumor vessels (which are more "leaky" than normal vessels) using intravital microscopy (8). Smaller QD are under active development, and we are exploring their biodistribution in mice. Although they are unlikely to escape recognition and clearance by the RES per se, they may extravasate more quickly, avoid RES sequestration, and thus might be able to reach tissue targets in higher amounts. Rather than being stored in RES indefinitely, smaller QD may also be cleared from the organism by renal excretion. This is preferable, as it is not known whether toxicity might be caused by residual QD in the liver and spleen.

To assess the stability of the radiolabeled QD, biodistribution studies of the 2 most likely degradation productsfree ${ }^{64} \mathrm{Cu}$ and DOTA chelated ${ }^{64} \mathrm{Cu}$-were performed. 


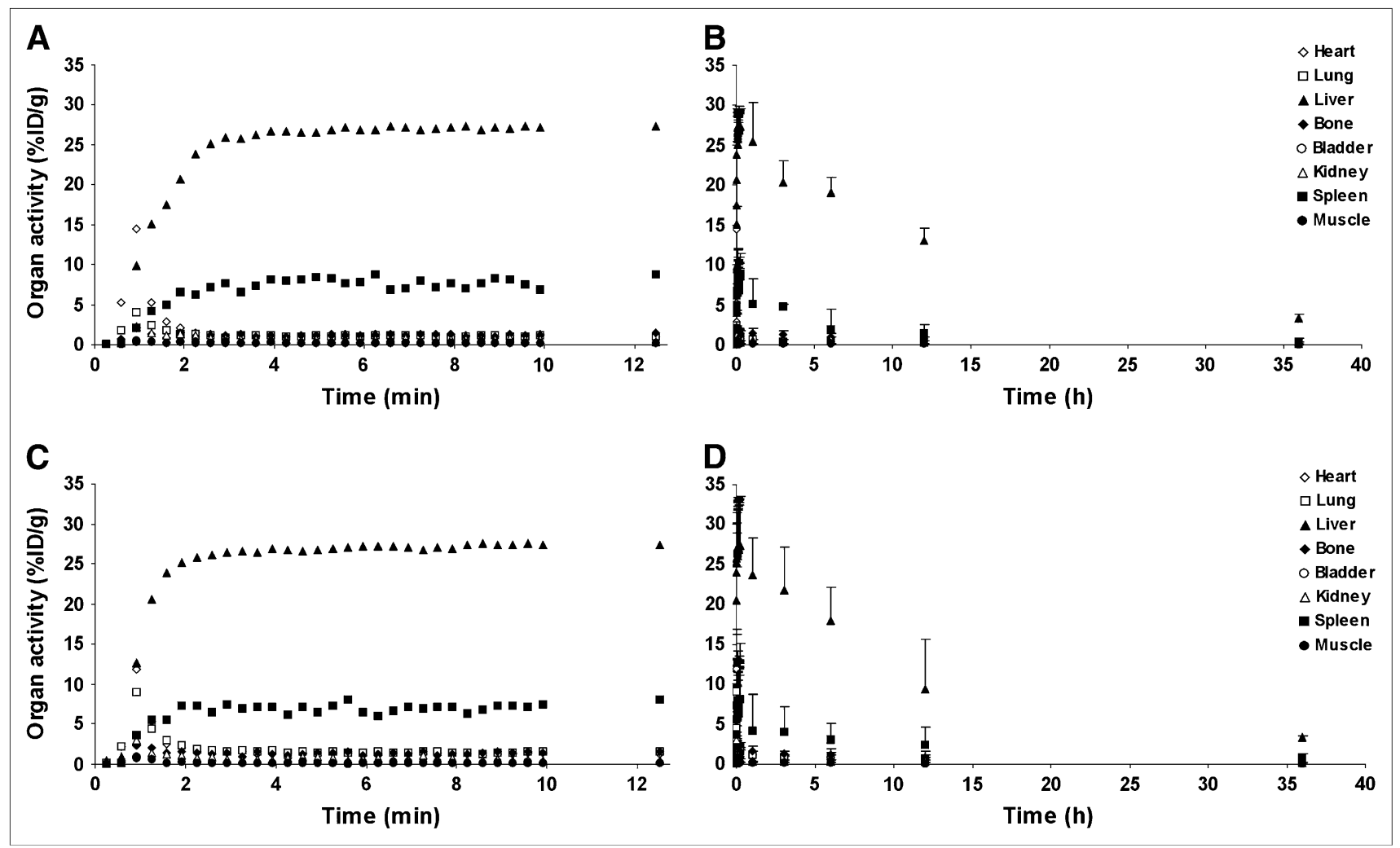

FIGURE 4. Image-based in vivo biodistribution of ${ }^{64} \mathrm{Cu}$-labeled, unpegylated QD525 (A and B) and QD800 (C and D) as measured by $\mathrm{ROI}$ analysis of microPET datasets. During dynamic image acquisition, $5.55 \mathrm{MBq}$ of radiolabeled QD were injected into tail vein of nude mice $(n=4)$. Images were acquired dynamically in 10-s frames for the first 10 min and one 5-min frame thereafter (A and C; error bars are omitted for better visibility). Mice were reimaged in 5-min static acquisitions at 1, 3, 6, 12, and 36 h (B and D), coregistered with microCT images, and AMIDE image analysis software was used to obtain organ activity information. Mean and $\mathrm{SD}$ of \%ID/g have not been corrected for physical decay.

Clearance routes were expectedly different from those of radiolabeled QD for both tracers, with kidney/bladder being the exclusive route of excretion for DOTA-complexed ${ }^{64} \mathrm{Cu}$ and a significant route of excretion for free ${ }^{64} \mathrm{Cu}$ as well. When assuming all kidney/bladder activity measured in the QD biodistributions to be attributable to free ${ }^{64} \mathrm{Cu}$ (or DOTA- ${ }^{64} \mathrm{Cu}$, respectively), a maximum of $2.8 \%-3 \%$ free ${ }^{64} \mathrm{Cu}$ (or of $0.04 \%$ DOTA $-{ }^{64} \mathrm{Cu}$ ) could be contained in each of our samples. However, the actual amounts are likely to be lower. At least part of the activity in kidney/bladder is likely to be from QD activity, rather than free ${ }^{64} \mathrm{Cu}$ or DOTA- ${ }^{64} \mathrm{Cu}$. Although no formal serum stability studies were performed in this work, the differences in biodistributions suggest good stability of the labeled QD in vivo. Following the same line of argumentation as above, the differences between biodistribution of QD and of both ${ }^{64} \mathrm{Cu}$ and DOTA- ${ }^{64} \mathrm{Cu}$ suggest that a minimum of $90 \%$ of radioactivity is bound at $12 \mathrm{~h}$.

There is a small chance that the presence of DOTA on the QD could alter the biodistribution of the particle. This appears relatively unlikely, as DOTA does not add significant size (given the relative dimensions of DOTA and QD) or charge (given that the PEG covering the QD surface is already extremely polar). However, it would be interesting in future studies to directly label the QD with the radioac- tive compound or to fabricate QD from isotopes that can be used for imaging. Although it was not the purpose of this study to investigate $\mathrm{CdSe} / \mathrm{ZnS}$ QD toxicity, and future detailed work is necessary on this topic, no evidence of acute toxicity was observed during and after the experiments performed here.

For a useful imaging agent, the amount of measurable agent at the target (e.g., a tumor) should be considerably higher than that in the background. For a high target-tobackground ratio, 3 criteria are relevant: rapid diffusion/ transport of the agent to the target site, high specificity and affinity of target binding, and effective clearance of unbound agent from the background. However, clearance should be slower than the time needed to reach and bind the target to enable a significant portion of the agent to reach the target site. Unfortunately, the rapid RES blood clearance of commercially available QD formulations observed in this study allows little time for a significant portion of the particles to reach potential targets. Although targets on the vascular endothelial surface may still be accessible to some degree (and, indeed, all reports of in vivo targeting of QD published to date have used targets on the vascular endothelial surface $(3,5,6))$, it is unlikely that targets requiring extravasation to the interstitial space (e.g., targets expressed on the surface of 

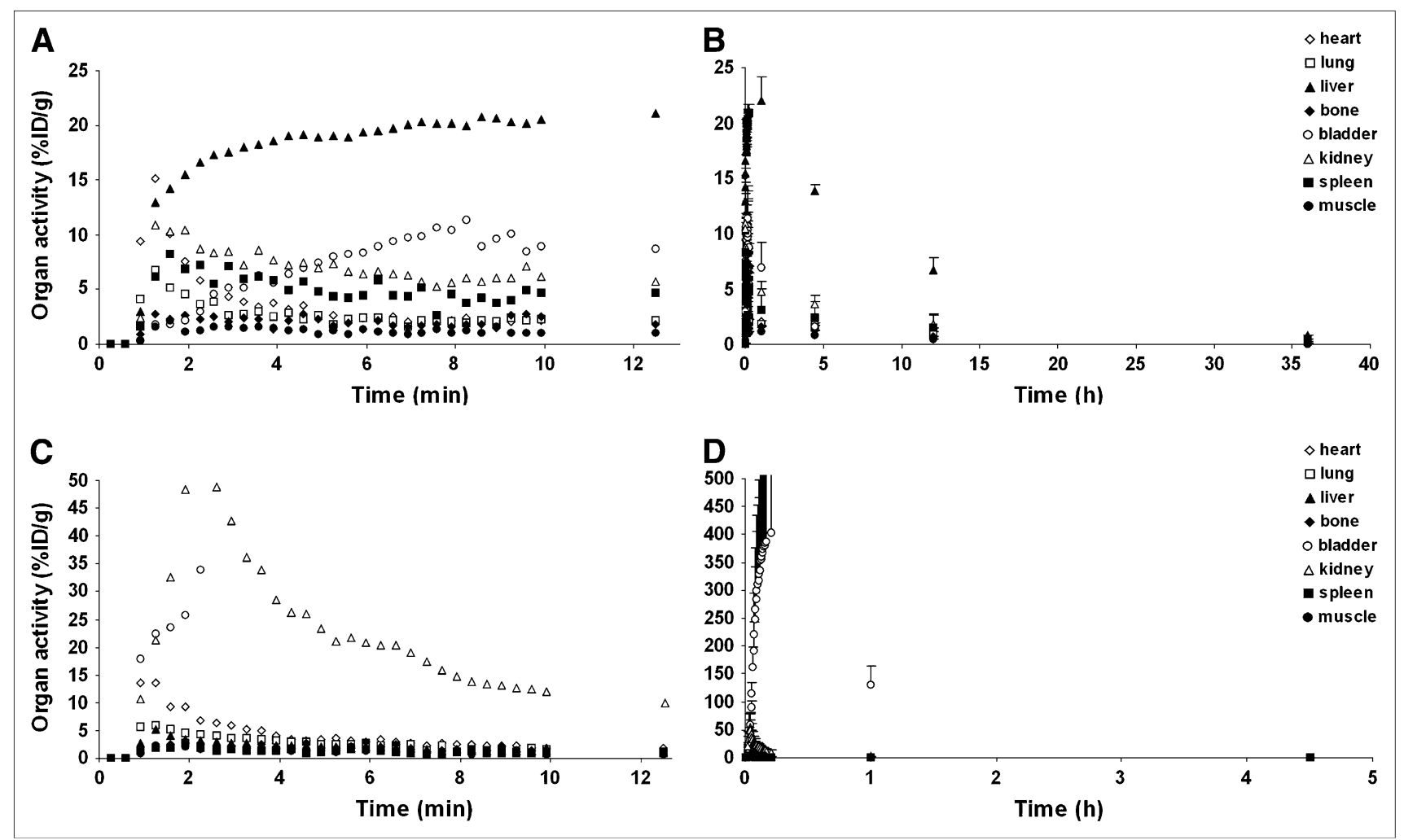

FIGURE 5. Image-based in vivo biodistribution of ${ }^{64} \mathrm{Cu}(\mathrm{A}$ and $\mathrm{B})$ and $\mathrm{DOTA}-{ }^{64} \mathrm{Cu}(\mathrm{C}$ and $\mathrm{D})$ as measured by ROI analysis of PET datasets. During dynamic image acquisition, $5.55 \mathrm{MBq}$ of the respective tracer were injected into tail vein of nude mice $(n=2)$. Images were acquired dynamically in 10-s frames for the first $10 \mathrm{~min}$ and one 5-min frame thereafter (A and C; error bars are omitted for better visibility). Mice were reimaged in 5-min static acquisitions at 1, 3, 6, 12, and $36 \mathrm{~h}(\mathrm{~B}$ and $\mathrm{D})$, coregistered with microCT images, and AMIDE image analysis software was used to obtain organ activity information. Mean and SD of \%ID/g have not been corrected for physical decay.

a tumor) will be reached. In addition to the fast clearance, the identical biodistribution of large and small QD implies absence of extravasation of the specific particles tested here. However, absence of extravasation should be verified in future studies using intravital microscopy. There are 2 possible approaches to address this dilemma. Circulation time could be increased (e.g., by designing better surface coats) and the time required for QD to reach their target could be reduced (by increased extravasation of smaller particles or surface coats that encourage transport through vascular endothelium). Clearly, the composition of surface coatings protecting nanoparticles from RES clearance needs further refinement and should be a focus of intense research effort.

Lastly, we observed comparable results between well counting and microPET-based analysis of organ activity, as expected from studies showing that PET accurately measures radioactivity in phantoms (19). However, absolute values varied somewhat between the methods, and SD were nonnegligible due to the small number of animals $(n=3)$ tested at each time point and large interindividual variations in uptake. In contrast to ex vivo counting, where large variations existed between time points (reflecting interindividual differences between the various animals that were sacrificed at each time point), results from in vivo imaging- based analysis were more consistent between time points, as the same animals were being repetitively imaged. Partialvolume effects (averaging of counts over voxels that contain both hot and cold areas) should introduce a systematic bias to lower values in small organs (relative to the resolution of the scanner). In large organs, partial-volume effects can be circumvented by placing the ROI within the organ, away from organ borders. Small organs may not have areas that are unaffected by partial-volume effects. In our study, the organs that primarily might be at risk are bone and spleen. However, values obtained by ex vivo measurements and PET for bone and spleen were quite similar. In the light of our data, the bias toward lower uptake introduced by partial-volume effects appears to be relatively small (in fact, not significant) when compared with other sources of variation (interindividual variations).

Dynamic imaging allowed for the generation of timeactivity curves for organs of interest, which is of great help in understanding early pharmacokinetics of an agent. For example, in our study, conventional biodistribution would have been unable to distinguish between pegylated and unpegylated particles, as the relevant pharmacokinetic difference occurred before the first time point at which animals were sacrificed. 
Using image-based analysis of organ activity reduced the number of necessary mice by $80 \%$, resulting in a significant reduction of animal use as well as of cost for animal acquisition and housing. Radiotracer cost was slightly higher with imaging, because more activity is needed for imaging than for well counting, but this did not outweigh the gains of lower animal cost. After an initial time commitment for familiarization with the software, image-based analysis consumed approximately 50\% less time than well counting.

Although QD are fluorescence imaging agents, preliminary studies from our laboratory show that fluorescence imaging systems significantly underestimate QD mass amounts in living animals, especially when they are located in deeper lying tissues, and are currently not robust enough for biodistribution purposes (data not shown).

\section{CONCLUSION}

Knowledge of quantitative biodistribution is of paramount importance in any diagnostic or therapeutic application of nanomaterials in living animals. In nude mice, both well counting and microPET show rapid uptake of radiolabeled QD by liver and spleen. Size of the particles has no influence on biodistribution within the range that was tested here. Pegylated QD show slightly slower uptake into liver and spleen (6 vs. $2 \mathrm{~min}$ ) and are taken up into bone at low levels in addition to liver and spleen. No evidence of biologic clearance from RES organs was observed. Rapid RES clearance of QD may impair their usefulness for in vivo fluorescence imaging, and smaller QD may help to solve this problem.

\section{ACKNOWLEDGMENTS}

This study was supported in part by NCI ICMIC P50 CA114747, NCI CCNE U54 CA119367, and NIBIB BRP 5-RO1-EB000312 grants. We are indebted to Shay Keren, Yuval Ebenstein, Olivier Gheysens, Gopalakrishnan Sundaresan, Jianqing Jack Li, Christophe DeRoose, and Andreas M. Loening for helpful discussions.

\section{REFERENCES}

1. Michalet X, Pinaud FF, Bentolila LA, et al. Quantum dots for live cells, in vivo imaging, and diagnostics. Science. 2005;307:538-544.
2. Gao X, Yang L, Petros JA, Marshall FF, Simons JW, Nie S. In vivo molecular and cellular imaging with quantum dots. Curr Opin Biotechnol. 2005;16: 63-72.

3. Gao X, Cui Y, Levenson RM, Chung LW, Nie S. In vivo cancer targeting and imaging with semiconductor quantum dots. Nat Biotechnol. 2004;22:969-976.

4. Chu TC, Shieh F, Lavery LA, et al. Labeling tumor cells with fluorescent nanocrystal-aptamer bioconjugates. Biosens Bioelectron. 2006;21:1859-1866.

5. Akerman ME, Chan WC, Laakkonen P, Bhatia SN, Ruoslahti E. Nanocrystal targeting in vivo. Proc Natl Acad Sci USA. 2002;99:12617-12621.

6. Cai W, Shin DW, Chen K, et al. Peptide-labeled near-infrared quantum dots for imaging tumor vasculature in living subjects. Nano Lett. 2006;6:669-676.

7. Bharali DJ, Lucey DW, Jayakumar H, Pudavar HE, Prasad PN. Folate-receptormediated delivery of InP quantum dots for bioimaging using confocal and twophoton microscopy. J Am Chem Soc. 2005;127:11364-11371.

8. Stroh M, Zimmer JP, Duda DG, et al. Quantum dots spectrally distinguish multiple species within the tumor milieu in vivo. Nat Med. 2005;11:678-682.

9. Allen C, Dos Santos N, Gallagher R, et al. Controlling the physical behavior and biological performance of liposome formulations through use of surface grafted poly(ethylene glycol). Biosci Rep. 2002;22:225-250.

10. Owens DE III, Peppas NA. Opsonization, biodistribution, and pharmacokinetics of polymeric nanoparticles. Int J Pharm. 2006;307:93-102.

11. Parungo CP, Ohnishi S, Kim SW, et al. Intraoperative identification of esophageal sentinel lymph nodes with near-infrared fluorescence imaging. $J$ Thorac Cardiovasc Surg. 2005;129:844-850.

12. Soltesz EG, Kim S, Kim SW, et al. Sentinel lymph node mapping of the gastrointestinal tract by using invisible light. Ann Surg Oncol. 2006;13:386-396.

13. Zimmer JP, Kim SW, Ohnishi S, Tanaka E, Frangioni JV, Bawendi MG. Size series of small indium arsenide-zinc selenide core-shell nanocrystals and their application to in vivo imaging. J Am Chem Soc. 2006;128:2526-2527.

14. Thorne RG, Nicholson C. In vivo diffusion analysis with quantum dots and dextrans predicts the width of brain extracellular space. Proc Natl Acad Sci USA. 2006;103:5567-5572.

15. Ballou B, Lagerholm BC, Ernst LA, Bruchez MP, Waggoner AS. Noninvasive imaging of quantum dots in mice. Bioconjug Chem. 2004;15:79-86.

16. Comsa DC, Farrell TJ, Patterson MS. Quantification of bioluminescence images of point source objects using diffusion theory models. Phys Med Biol. 2006;51: 3733-3746.

17. So MK, Xu C, Loening AM, Gambhir SS, Rao J. Self-illuminating quantum dot conjugates for in vivo imaging. Nat Biotechnol. 2006;24:339-343.

18. Tseng JR, Dandekar M, Subbarayan M, et al. Reproducibility of 3'-deoxy$3^{\prime}-{ }^{18} \mathrm{~F}$-fluorothymidine microPET studies in tumor xenografts in mice. $\mathrm{J} \mathrm{Nucl}$ Med. 2005;46:1851-1857.

19. Knoess C, Siegel S, Smith A, et al. Performance evaluation of the microPET R4 PET scanner for rodents. Eur J Nucl Med Mol Imaging. 2003;30:737-747.

20. Loening AM, Gambhir SS. AMIDE: a free software tool for multimodality medical image analysis. Mol Imaging. 2003;2:131-137.

21. Moghimi SM, Szebeni J. Stealth liposomes and long circulating nanoparticles: critical issues in pharmacokinetics, opsonization and protein-binding properties. Prog Lipid Res. 2003;42:463-478.

22. Laverman P, Boerman OC, Oyen WJG, Corstens FHM, Storm G. In vivo applications of PEG liposomes: unexpected observations. Crit Rev Ther Drug Carrier Syst. 2001;18:551-566.

23. Laverman P, Brouwers AH, Dams ET, et al. Preclinical and clinical evidence for disappearance of long-circulating characteristics of polyethylene glycol liposomes at low lipid dose. J Pharmacol Exp Ther. 2000;293:996-1001.

24. Laverman P, Carstens MG, Boerman OC, et al. Factors affecting the accelerated blood clearance of polyethylene glycol-liposomes upon repeated injection. J Pharmacol Exp Ther. 2001;298:607-612. 